\title{
Nonlinear sequential fractional differential equations with nonlocal boundary conditions
}

\author{
Nazim I Mahmudov, Muath Awadalla and Kanda Abuassba*
}

\section{"Correspondence:}

kinda.joudeh@gmail.com Eastern Mediterranean University, Gazimagusa, T.R. North Cyprus, Mersin 10, Turkey

\begin{abstract}
This article develops the existence theory for sequential fractional differential equations involving Caputo fractional derivative of order $1<\alpha<2$ with nonlocal integral boundary conditions. Examples are given to demonstrate applications of our results.

MSC: $34 \mathrm{~A} 08 ; 34 \mathrm{~B} 10$

Keywords: Caputo fractional derivative; sequential fractional derivative; existence; fixed point
\end{abstract}

\section{Introduction}

Fractional calculus is a natural extension of ordinary calculus, where integrals and derivatives are defined for arbitrary real orders. Since the 17th century, when fractional calculus was born, several different derivatives have been introduced: Riemann-Liouville, Hadamard, Grunwald-Letnikov, and Caputo, just to mention a few [1-3]. The choice of an appropriate fractional derivative depends on the considered system, and for this reason, we find a large number of publications devoted to different fractional operators. Nowadays, fractional-order differential and integral operators, which are nonlocal in nature, appear in mathematical models of many real world phenomena such as anomalous diffusion, ecological effects, blood flow issues, spreading of disease, control processes, etc. A wide range of application of fractional calculus has motivated many researchers to develop the theoretical aspects of this branch of modern analysis. Recently, many authors have presented new types of fractional operators, which generalize the Riemann-Liouville, Caputo and Hadamard fractional operators. For theoretical development and application details, we refer the reader to [3-18] and the references cited therein. The theory of fractionalorder differential equations involving different kinds of boundary conditions has been a field of interest in pure and applied sciences. In addition to the classical two-point boundary conditions, great attention is paid to nonlocal multipoint and integral boundary conditions. Nonlocal conditions are used to describe certain features of physical, chemical or other processes occurring in the internal positions of the given region, while integral boundary conditions provide a plausible and practical approach to modeling the problems of blood flow. For more details and explanation, see, for instance [1, 2]. Some recent results on fractional-order boundary value problems (BVPs) can be found in a series of papers [19-29] and the references cited therein. Sequential fractional differential equa-

(c) The Author(s) 2017. This article is distributed under the terms of the Creative Commons Attribution 4.0 International License (http://creativecommons.org/licenses/by/4.0/), which permits unrestricted use, distribution, and reproduction in any medium, provided you give appropriate credit to the original author(s) and the source, provide a link to the Creative Commons license, and indicate if changes were made. 
tions have also received considerable attention, for instance see [19-25]. To the best of our knowledge, the study of sequential fractional differential equations supplemented with four point nonlocal integral fractional boundary conditions has yet to be initiated.

Motivated by the above papers we establish the existence of solutions for the following nonlinear sequential fractional differential equation subject to nonlocal fractional integral conditions

$$
\left\{\begin{array}{l}
\left({ }^{c} D^{\alpha}+k^{c} D^{\alpha-1}\right) u(t)=f\left(t, u(t),{ }^{c} D^{\alpha-1} u(t)\right), \quad 1<\alpha<2,0 \leq t \leq T, \\
\alpha_{1} u(\eta)+\beta_{1} u(T)=\gamma_{1} \int_{0}^{\xi} u(s) d s+\varepsilon_{1}, \\
\alpha_{2}{ }^{c} D^{\alpha-1} u(\eta)+\beta_{2}{ }^{c} D^{\alpha-1} u(T)=\gamma_{2} \int_{\zeta}^{T} u(s) d s+\varepsilon_{2},
\end{array}\right.
$$

where ${ }^{c} D^{\alpha}$ is the standard Caputo fractional derivative of order $\alpha, 0 \leq \eta \leq T, 0<\xi<\zeta<T$, $\alpha_{1}, \alpha_{2}, \beta_{1}, \beta_{2}, \gamma_{1}, \gamma_{2}, \varepsilon_{1}, \varepsilon_{2} \in \mathbb{R}$.

Notations:

$$
\begin{aligned}
& \mathcal{R}=\mathcal{R}_{1}+\mathcal{R}_{2}, \quad \mathcal{R}_{1}=\mathcal{R}_{1}^{*}+\frac{T^{\alpha-1}\left(1-e^{-k T}\right)}{k \Gamma(\alpha)}, \\
& \mu_{1}(t)=\left(\frac{\varepsilon_{2} \rho}{q z}+\frac{\varepsilon_{1} \rho \sigma}{q^{2} z}+\frac{\varepsilon_{1}}{q}\right) e^{-k t}-\left(\frac{\varepsilon_{2}}{z}+\frac{\varepsilon_{1} \sigma}{q z}\right), \quad \mu_{2}(t)=\left(\frac{\gamma_{1} \rho \sigma}{q^{2} z}+\frac{\gamma_{1}}{q}\right) e^{-k t}-\frac{\gamma_{1} \sigma}{q z}, \\
& \mu_{3}(t)=\left(\frac{\gamma_{2} \rho}{q z}\right) e^{-k t}-\left(\frac{\gamma_{2}}{z}\right), \quad \mu_{4}(t)=\left(\frac{\alpha_{1} \sigma}{q z}\right)-\left(\frac{\alpha_{1} \rho \sigma}{q^{2} z}+\frac{\alpha_{1}}{q}\right) e^{-k t}, \\
& \mu_{5}(t)=\left(\frac{\beta_{1} \sigma}{q z}\right)-\left(\frac{\beta_{1} \rho \sigma}{q^{2} z}+\frac{\beta_{1}}{q}\right) e^{-k t}, \quad \mu_{6}(t)=\frac{\alpha_{2}}{z}-\left(\frac{\alpha_{2} \rho}{q z}\right) e^{-k t} \\
& \mu_{7}(t)=\frac{\beta_{2}}{z}-\left(\frac{\beta_{2} \rho}{q z}\right) e^{-k t}, \quad \mu_{8}(t)=\left(\frac{\alpha_{2} k \rho}{q z}\right) e^{-k t}-\frac{\alpha_{2} k}{z} \\
& \mu_{9}(t)=\left(\frac{\beta_{2} k \rho}{q z}\right) e^{-k t}-\frac{\beta_{2} k}{z},
\end{aligned}
$$

where

$$
\begin{aligned}
q= & \left(\alpha_{1} e^{-k \eta}+\beta_{1} e^{-k T}-\gamma_{1}\left(1-e^{-k \xi}\right)\right), \quad \rho=\left(\alpha_{1}+\beta_{1}-\gamma_{1} \xi\right), \\
\sigma= & \left(\frac{\gamma_{2}}{k}\left(e^{-k \zeta}-e^{-k T}\right)+\frac{k \alpha_{2}}{\Gamma(2-\alpha)} \int_{0}^{\eta}(\eta-s)^{1-\alpha} e^{-k s} d s\right. \\
& \left.+\frac{k \beta_{2}}{\Gamma(2-\alpha)} \int_{0}^{T}(T-s)^{1-\alpha} e^{-k s} d s\right), \\
z= & \gamma_{2}(T-\zeta)-\frac{\sigma \rho}{q},
\end{aligned}
$$

with

$$
\begin{aligned}
& K_{1}(t, x)=\frac{1}{\Gamma(\alpha-1)} \int_{s}^{t} e^{-k(t-s)}(s-x)^{\alpha-2} d s, \\
& \int_{0}^{t} K_{1}(t, x) h(x) d x=\int_{0}^{t} e^{-k(t-s)} I^{\alpha-1} h(s) d s, \\
& K_{2}(t, s)=\frac{1}{\Gamma(2-\alpha)} \int_{s}^{t}(t-x)^{1-\alpha} K_{1}(x, s) d x,
\end{aligned}
$$




$$
\begin{aligned}
& \frac{1}{\Gamma(2-\alpha)} \int_{0}^{\eta}(\eta-s)^{1-\alpha} \int_{0}^{s} e^{-k(s-x)} I^{\alpha-1} h(x) d x d s \\
& \quad=\frac{1}{\Gamma(2-\alpha)} \int_{0}^{\eta}(\eta-s)^{1-\alpha} \int_{0}^{s} K_{1}(s, x) h(x) d x d s \\
& \quad=\frac{1}{\Gamma(2-\alpha)} \int_{0}^{\eta}\left(\int_{x}^{\eta}(\eta-s)^{1-\alpha} K_{1}(s, x) d s\right) h(x) d x=\int_{0}^{\eta} K_{2}(\eta, x) h(x) d x .
\end{aligned}
$$

Lemma 1 Let $h \in C([0, T], \mathbb{R})$. The solution of the sequential $F D E$

$$
\left\{\begin{array}{l}
\left({ }^{c} D^{\alpha}+k^{c} D^{\alpha-1}\right) u(t)=h(t), \quad 1<\alpha \leq 2,0 \leq t \leq T, \\
\alpha_{1} u(\eta)+\beta_{1} u(T)=\gamma_{1} \int_{0}^{\xi} u(s) d s+\varepsilon_{1} \\
\alpha_{2}{ }^{c} D^{\alpha-1} u(\eta)+\beta_{2}{ }^{c} D^{\alpha-1} u(T)=\gamma_{2} \int_{\zeta}^{T} u(s) d s+\varepsilon_{2},
\end{array}\right.
$$

has the following representation:

$$
\begin{aligned}
u(t)= & \mu_{1}(t)+\int_{0}^{t} K_{1}(t, x) h(s) d s+\mu_{2}(t) \int_{0}^{\xi} \int_{0}^{s} K_{1}(s, x) h(x) d x d s \\
& +\mu_{3}(t) \int_{\zeta}^{T} \int_{0}^{s} K_{1}(s, x) h(x) d x d s+\mu_{4}(t) \int_{0}^{\eta} K_{1}(\eta, x) h(x) d x \\
& +\mu_{5}(t) \int_{0}^{T} K_{1}(T, x) h(x) d x+\mu_{6}(t) \int_{0}^{\eta} h(x) d x+\mu_{7}(t) \int_{0}^{T} h(x) d x \\
& +\mu_{8}(t) \int_{0}^{\eta} K_{2}(\eta, x) h(x) d x+\mu_{9}(t) \int_{0}^{T} K_{2}(T, x) h(x) d x .
\end{aligned}
$$

The function $u(t)$ defined above is a solution of (2).

Proof Solving the sequential equation $\left({ }^{c} D^{\alpha}+k^{c} D^{\alpha-1}\right) u(t)=h(t)$, we get

$$
u(t)=A_{0} e^{-k t}+A_{1}+\int_{0}^{t} e^{-k(t-s)} I^{\alpha-1} h(s) d s
$$

Now, we need to find the constants $A_{0}$ and $A_{1}$. Taking the fractional derivative of $u(t)$ we get ${ }^{c} D^{\alpha-1} u(t)=\frac{-k A_{0}}{\Gamma(2-\alpha)} \int_{0}^{t}(t-s)^{1-\alpha} e^{-k s} d s+\frac{1}{\Gamma(2-\alpha)} \int_{0}^{t}(t-s)^{1-\alpha}\left(I^{\alpha-1} h(s)-k \int_{0}^{s} e^{-k(s-x)} I^{\alpha-1} \times\right.$ $h(x) d x) d s$. The first boundary condition of (2) gives

$$
\begin{aligned}
A_{0}\left(\alpha_{1} e^{-k \eta}+\beta_{1} e^{-k T}-\gamma_{1}\left(1-e^{-k \xi}\right)\right)+A_{1}\left(\alpha_{1}+\beta_{1}-\gamma_{1} \xi\right) \\
=\varepsilon_{1}+\gamma_{1} \int_{0}^{\xi} \int_{0}^{s} e^{-k(s-x)} I^{\alpha-1} h(x) d x d s \\
\quad-\alpha_{1} \int_{0}^{\eta} e^{-k(\eta-s)} I^{\alpha-1} h(s) d s-\beta_{1} \int_{0}^{T} e^{-k(T-s)} I^{\alpha-1} h(s) d s .
\end{aligned}
$$

From the second boundary condition of (2), we get

$$
\begin{aligned}
& A_{0}\left(\frac{\gamma_{2}}{k}\left(e^{-k \zeta}-e^{-k T}\right)+\frac{k \alpha_{2}}{\Gamma(2-\alpha)} \int_{0}^{\eta}(\eta-s)^{1-\alpha} e^{-k s} d s+\frac{k \beta_{2}}{\Gamma(2-\alpha)} \int_{0}^{T}(T-s)^{1-\alpha} e^{-k s} d s\right) \\
& \quad+A_{1} \gamma_{2}(T-\zeta)
\end{aligned}
$$




$$
\begin{aligned}
= & -\varepsilon_{2}-\gamma_{2} \int_{\zeta}^{T} \int_{0}^{s} e^{-k(s-x)} I^{\alpha-1} h(x) d x d s+\frac{\alpha_{2}}{\Gamma(2-\alpha)} \int_{0}^{\eta}(\eta-s)^{1-\alpha} I^{\alpha-1} h(s) d s \\
& -\frac{k \alpha_{2}}{\Gamma(2-\alpha)} \int_{0}^{\eta}(\eta-s)^{1-\alpha}\left(\int_{0}^{s} e^{-k(s-x)} I^{\alpha-1} h(x) d x\right) d s \\
& +\frac{\beta_{2}}{\Gamma(2-\alpha)} \int_{0}^{T}(T-s)^{1-\alpha} I^{\alpha-1} h(s) d s \\
& -\frac{k \beta_{2}}{\Gamma(2-\alpha)} \int_{0}^{T}(T-s)^{1-\alpha}\left(\int_{0}^{s} e^{-k(s-x)} I^{\alpha-1} h(x) d x\right) d s .
\end{aligned}
$$

A simultaneous solution of (4) and (5) leads to

$$
\begin{aligned}
A_{0}= & \left(\frac{\varepsilon_{2} \rho}{q z}+\frac{\varepsilon_{1} \rho \sigma}{q^{2} z}+\frac{\varepsilon_{1}}{q}\right)+\left(\frac{\gamma_{1} \rho \sigma}{q^{2} z}+\frac{\gamma_{1}}{q}\right) \int_{0}^{\xi} \int_{0}^{s} K_{1}(s, x) h(x) d x d s \\
& -\left(\frac{\alpha_{1} \rho \sigma}{q^{2} z}+\frac{\alpha_{1}}{q}\right) \int_{0}^{\eta} K_{1}(\eta, x) h(x) d x \\
& -\left(\frac{\beta_{1} \rho \sigma}{q^{2} z}+\frac{\beta_{1}}{q}\right) \int_{0}^{T} K_{1}(T, x) h(x) d x+\left(\frac{\gamma_{2} \rho}{q z}\right) \int_{\zeta}^{T} \int_{0}^{s} K_{1}(s, x) h(x) d x d s \\
& -\left(\frac{\alpha_{2} \rho}{q z}\right) \int_{0}^{\eta} h(x) d x+\left(\frac{\alpha_{2} k \rho}{q z}\right) \int_{0}^{\eta} K_{2}(\eta, x) h(x) d x \\
& -\left(\frac{\beta_{2} \rho}{q z}\right) \int_{0}^{T} h(x) d x+\left(\frac{\beta_{2} k \rho}{q z}\right) \int_{0}^{T} K_{2}(T, x) h(x) d x . \\
A_{1}= & \left(-\frac{\gamma_{1} \sigma}{q z}\right) \int_{0}^{\xi} \int_{0}^{s} K_{1}(s, x) h(x) d x d s+\left(\frac{\alpha_{1} \sigma}{q z}\right) \int_{0}^{\eta} K_{1}(\eta, x) h(x) d x \\
& +\left(\frac{\beta_{1} \sigma}{q z}\right) \int_{0}^{T} K_{1}(T, x) h(x) d x-\frac{\varepsilon_{2}}{z}-\frac{\varepsilon_{1} \sigma}{q z}-\left(\frac{\gamma_{2}}{z}\right) \int_{\zeta}^{T} \int_{0}^{s} K_{1}(s, x) h(x) d x d s \\
& +\frac{\alpha_{2}}{z} \int_{0}^{\eta} h(x) d x \\
& -\frac{\alpha_{2} k}{z} \int_{0}^{\eta} K_{2}(\eta, x) h(x) d x+\frac{\beta_{2}}{z} \int_{0}^{T} h(x) d x-\frac{\beta_{2} k}{z} \int_{0}^{T} K_{2}(T, x) h(x) d x .
\end{aligned}
$$

We substitute (6) and (7) in (3) to get the desired solution,

$$
\begin{aligned}
u(t)= & \underbrace{\left[\left(\frac{\varepsilon_{2} \rho}{q z}+\frac{\varepsilon_{1} \rho \sigma}{q^{2} z}+\frac{\varepsilon_{1}}{q}\right) e^{-k t}-\left(\frac{\varepsilon_{2}}{z}+\frac{\varepsilon_{1} \sigma}{q z}\right)\right]}_{\mu_{1}(t)}+\int_{0}^{t} K_{1}(t, x) h(s) d s \\
& +\underbrace{\left[\left(\frac{\gamma_{1} \rho \sigma}{q^{2} z}+\frac{\gamma_{1}}{q}\right) e^{-k t}-\frac{\gamma_{1} \sigma}{q z}\right]}_{\mu_{2}(t)} \int_{0}^{\xi} \int_{0}^{s} K_{1}(s, x) h(x) d x d s \\
& +\underbrace{\left[\left(\frac{\gamma_{2} \rho}{q z}\right) e^{-k t}-\left(\frac{\gamma_{2}}{z}\right)\right]}_{\mu_{3}(t)} \int_{\zeta}^{T} \int_{0}^{s} K_{1}(s, x) h(x) d x d s \\
& +\underbrace{\left[\left(\frac{\alpha_{1} \sigma}{q z}\right)-\left(\frac{\alpha_{1} \rho \sigma}{q^{2} z}+\frac{\alpha_{1}}{q}\right) e^{-k t}\right]}_{\mu_{4}(t)} \int_{0}^{\eta} K_{1}(\eta, x) h(x) d x
\end{aligned}
$$




$$
\begin{aligned}
& +\underbrace{\left[\left(\frac{\beta_{1} \sigma}{q z}\right)-\left(\frac{\beta_{1} \rho \sigma}{q^{2} z}+\frac{\beta_{1}}{q}\right) e^{-k t}\right]}_{\mu_{5}(t)} \int_{0}^{T} K_{1}(T, x) h(x) d x \\
& +\underbrace{\left[\frac{\alpha_{2}}{z}-\left(\frac{\alpha_{2} \rho}{q z}\right) e^{-k t}\right]}_{\mu_{6}(t)} \int_{0}^{\eta} h(x) d x+\underbrace{\left[\frac{\beta_{2}}{z}-\left(\frac{\beta_{2} \rho}{q z}\right) e^{-k t}\right]}_{\mu_{7}(t)} \int_{0}^{T} h(x) d x \\
& +\underbrace{\left[\left(\frac{\alpha_{2} k \rho}{q z}\right) e^{-k t}-\frac{\alpha_{2} k}{z}\right]}_{\mu_{8}(t)} \int_{0}^{\eta} K_{2}(\eta, x) h(x) d x \\
& +\underbrace{\left[\left(\frac{\beta_{2} k \rho}{q z}\right)_{e^{-k t}}-\frac{\beta_{2} k}{z}\right]}_{\mu_{9}(t)} \int_{0}^{T} K_{2}(T, x) h(x) d x .
\end{aligned}
$$

By direct computation the converse of the lemma holds. This completes the proof.

Lemma 2 For any $g, p \in C([0, T] ; \mathbb{R})$ we have

$$
\begin{aligned}
& \left|\int_{0}^{t} K_{1}(t, x) g(x) d x-\int_{0}^{t} K_{1}(t, x) p(x) d x\right| \leq \frac{t^{\alpha-1}}{k \Gamma(\alpha)}\left(1-e^{-k t}\right)\|g-p\|, \\
& \left|\int_{0}^{t} K_{2}(t, x) g(x) d x-\int_{0}^{t} K_{2}(t, x) p(x) d x\right| \leq \frac{t}{k}\left(1-e^{-k t}\right)\|g-p\| .
\end{aligned}
$$

\section{Main results}

Given $C_{\alpha-1}[0, T]=\left\{u \in C[0, T]:{ }^{c} D^{\alpha-1} u \in C[0, T]\right\}$. Let $\Psi=\left(C_{\alpha-1}[0, T],\|\cdot\|_{\alpha-1}\right)$ denote the Banach space of all continuous functions from $[0, T]$ to $\mathbb{R}$, endowed with the norm defined by $\|u\|_{\alpha-1}=\sup _{0 \leq t \leq T}|u(t)|+\sup _{0 \leq t \leq T}\left|{ }^{c} D^{\alpha-1} u(t)\right|$.

Replacing $h(x)$ by $f\left(x, u(x),{ }^{c} D^{\alpha-1} u(x)\right)$ in Lemma 1 , an operator $\mathcal{F}: \Psi \rightarrow \Psi$ associated with problem (1) can be defined as

$$
\begin{aligned}
(\mathcal{F} u)(t)= & \mu_{1}(t)+\int_{0}^{t} K_{1}(t, x) f\left(x, u(x),{ }^{c} D^{\alpha-1} u(x)\right) d x \\
& +\mu_{2}(t) \int_{0}^{\xi} \int_{0}^{s} K_{1}(s, x) f\left(x, u(x),{ }^{c} D^{\alpha-1} u(x)\right) d x d s \\
& +\mu_{3}(t) \int_{\zeta}^{T} \int_{0}^{s} K_{1}(s, x) f\left(x, u(x),{ }^{c} D^{\alpha-1} u(x)\right) d x d s \\
& +\mu_{4}(t) \int_{0}^{\eta} K_{1}(\eta, x) f\left(x, u(x),{ }^{c} D^{\alpha-1} u(x)\right) d x \\
& +\mu_{5}(t) \int_{0}^{T} K_{1}(T, x) f\left(x, u(x),{ }^{c} D^{\alpha-1} u(x)\right) d x \\
& +\mu_{6}(t) \int_{0}^{\eta} f\left(x, u(x),{ }^{c} D^{\alpha-1} u(x)\right) d x \\
& +\mu_{7}(t) \int_{0}^{T} f\left(x, u(x),{ }^{c} D^{\alpha-1} u(x)\right) d x \\
& +\mu_{8}(t) \int_{0}^{\eta} K_{2}(\eta, x) f\left(x, u(x),{ }^{c} D^{\alpha-1} u(x)\right) d x \\
& +\mu_{9}(t) \int_{0}^{T} K_{2}(T, x) f\left(x, u(x),{ }^{c} D^{\alpha-1} u(x)\right) d x .
\end{aligned}
$$


Observe that

$$
\begin{aligned}
\left({ }^{c} D^{\alpha-1} \mathcal{F} u\right)(t)= & \left({ }^{c} D^{\alpha-1} \mu_{1}\right)(t)+\int_{0}^{t} f\left(x, u(x),{ }^{c} D^{\alpha-1} u(x)\right) d x \\
& -k \int_{0}^{t} K_{2}(t, x) f\left(x, u(x),{ }^{c} D^{\alpha-1} u(x)\right) d x \\
& +\left({ }^{c} D^{\alpha-1} \mu_{2}\right)(t) \int_{0}^{\xi} \int_{0}^{s} K_{1}(s, x) f\left(x, u(x),{ }^{c} D^{\alpha-1} u(x)\right) d x d s \\
& +\left({ }^{c} D^{\alpha-1} \mu_{3}\right)(t) \int_{\zeta}^{T} \int_{0}^{s} K_{1}(s, x) f\left(x, u(x),{ }^{c} D^{\alpha-1} u(x)\right) d x d s \\
& +\left({ }^{c} D^{\alpha-1} \mu_{4}\right)(t) \int_{0}^{\eta} K_{1}(\eta, x) f\left(x, u(x),{ }^{c} D^{\alpha-1} u(x)\right) d x \\
& +\left({ }^{c} D^{\alpha-1} \mu_{5}\right)(t) \int_{0}^{T} K_{1}(T, x) f\left(x, u(x),{ }^{c} D^{\alpha-1} u(x)\right) d x \\
& +\left({ }^{c} D^{\alpha-1} \mu_{6}\right)(t) \int_{0}^{\eta} f\left(x, u(x),{ }^{c} D^{\alpha-1} u(x)\right) d x \\
& +\left({ }^{c} D^{\alpha-1} \mu_{7}\right)(t) \int_{0}^{T} f\left(x, u(x),{ }^{c} D^{\alpha-1} u(x)\right) d x \\
& +\left({ }^{c} D^{\alpha-1} \mu_{8}\right)(t) \int_{0}^{\eta} K_{2}(\eta, x) f\left(x, u(x),{ }^{c} D^{\alpha-1} u(x)\right) d x \\
& \\
& \\
& \\
& \\
&
\end{aligned}
$$

For computational convenience, we set

$$
\begin{aligned}
\mathcal{R}_{1}= & \left(\frac{T^{\alpha-1}}{k \Gamma(\alpha)}\left(1-e^{-k T}\right)+\left\|\mu_{2}\right\| \int_{0}^{\xi} \frac{s^{\alpha-1}}{k \Gamma(\alpha)}\left(1-e^{-k s}\right) d s+\left\|\mu_{3}\right\| \int_{\zeta}^{T} \frac{s^{\alpha-1}}{k \Gamma(\alpha)}\left(1-e^{-k s}\right) d s\right. \\
& +\left\|\mu_{4}\right\| \frac{\eta^{\alpha-1}}{k \Gamma(\alpha)}\left(1-e^{-k \eta}\right)+\left\|\mu_{5}\right\| \frac{T^{\alpha-1}}{k \Gamma(\alpha)}\left(1-e^{-k T}\right) \\
& \left.+\eta\left\|\mu_{6}\right\|+T\left\|\mu_{7}\right\|+\left\|\mu_{8}\right\| \frac{\eta\left(1-e^{-k \eta}\right)}{k}+\left\|\mu_{9}\right\| \frac{T\left(1-e^{-k T}\right)}{k}\right), \\
\mathcal{R}_{2}= & \left(T+T\left(1-e^{-k T}\right)+\left\|{ }^{c} D^{\alpha-1} \mu_{2}\right\| \int_{0}^{\xi} \frac{s^{\alpha-1}}{k \Gamma(\alpha)}\left(1-e^{-k s}\right) d s\right. \\
& +\left\|{ }^{c} D^{\alpha-1} \mu_{3}\right\| \int_{\zeta}^{T} \frac{s^{\alpha-1}}{k \Gamma(\alpha)}\left(1-e^{-k s}\right) d s+\left\|{ }^{c} D^{\alpha-1} \mu_{4}\right\| \frac{\eta^{\alpha-1}}{k \Gamma(\alpha)}\left(1-e^{-k \eta}\right) \\
& +\left\|{ }^{c} D^{\alpha-1} \mu_{5}\right\| \frac{T^{\alpha-1}}{k \Gamma(\alpha)}\left(1-e^{-k T}\right)+\eta\left\|^{c} D^{\alpha-1} \mu_{6}\right\|+T\left\|{ }^{c} D^{\alpha-1} \mu_{7}\right\| \\
& \left.+\left\|{ }^{c} D^{\alpha-1} \mu_{8}\right\| \frac{\eta\left(1-e^{-k \eta}\right)}{k}+\left\|{ }^{c} D^{\alpha-1} \mu_{9}\right\| \frac{T\left(1-e^{-k T}\right)}{k}\right), \\
\mathcal{R}_{3}= & \mathcal{R}_{1}+\mathcal{R}_{2}-\frac{T^{\alpha-1}}{k \Gamma(\alpha)}\left(1-e^{-k T}\right)-T-T\left(1-e^{-k T}\right) .
\end{aligned}
$$

We deal with the existence and uniqueness of solutions for problem (1). Before stating and proving the main results, we introduce the following hypotheses: 
(A1) $f:[0, T] \times \mathbb{R} \times \mathbb{R} \rightarrow \mathbb{R}$ is jointly continuous.

(A2) There exists $L_{f}>0$ such that

$$
|f(t, u, \bar{u})-f(t, v, \bar{v})| \leq L_{f}(|u-v|+|\bar{u}-\bar{v}|), \quad \forall t \in[0, T], u, v, \bar{u}, \bar{v} \in \mathbb{R}
$$

(A3) There exists a function $y \in C\left([0, T], \mathbb{R}^{+}\right)$such that

$$
|f(t, u, v)| \leq y(t), \quad \forall(t, u, v) \in[0, T] \times \mathbb{R} \times \mathbb{R}
$$

(A4) There exist a function $g \in C\left([0, T], \mathbb{R}^{+}\right)$and a nondecreasing function $\chi: \mathbb{R}^{+} \rightarrow \mathbb{R}^{+}$such that

$$
|f(t, u, v)| \leq g(t) \chi(|u|+|v|), \quad \forall(t, u, v) \in[0, T] \times \mathbb{R} \times \mathbb{R}
$$

(A5) There exists a constant $W>0$ such that

$$
\frac{W}{\left\|\mu_{1}\right\|_{\alpha-1}+\|g\| \chi(r) \mathcal{R}}>1 .
$$

The next theorem gives us a uniqueness result.

Theorem 1 Assume that (A1), (A2) hold. If $L_{f} \mathcal{R}<1$, then the BVP(1) has a unique solution on $[0, T]$.

Proof Consider the operator $\mathcal{F}$ defined by (8) and define a ball $\mathfrak{B}_{r}=\left\{u \in C_{\alpha-1}[0, T]\right.$ : $\left.\|u\|_{\alpha-1} \leq r\right\}$ with $r \geq \frac{\left\|\mu_{1}\right\|_{\alpha-1}+N_{f} \mathcal{R}}{1-L_{f} \mathcal{R}}$, where $N_{f}=\sup _{0 \leq t \leq T}|f(t, 0,0)|$.

First we show that $\mathcal{F} \mathfrak{B}_{r} \subset \mathfrak{B}_{r}$. For any $u \in \mathfrak{B}_{r}, t \in[0, T]$, we have

$$
\begin{aligned}
|(\mathcal{F} u)(t)| \leq & \left\|\mu_{1}\right\|+\int_{0}^{t}\left|K_{1}(t, x)\right| f\left(x, u(x),{ }^{c} D^{\alpha-1} u(x)\right) \mid d x \\
& +\left\|\mu_{2}\right\| \int_{0}^{\xi} \int_{0}^{s}\left|K_{1}(s, x)\right| f\left(x, u(x),{ }^{c} D^{\alpha-1} u(x)\right) \mid d x d s \\
& +\left\|\mu_{3}\right\| \int_{\zeta}^{T} \int_{0}^{s}\left|K_{1}(s, x)\right|\left|f\left(x, u(x),{ }^{c} D^{\alpha-1} u(x)\right)\right| d x d s \\
& +\left\|\mu_{4}\right\| \int_{0}^{\eta}\left|K_{1}(\eta, x)\right|\left|f\left(x, u(x),{ }^{c} D^{\alpha-1} u(x)\right)\right| d x \\
& +\left\|\mu_{5}\right\| \int_{0}^{T}\left|K_{1}(T, x)\right| f\left(x, u(x),{ }^{c} D^{\alpha-1} u(x)\right) \mid d x \\
& +\left\|\mu_{6}\right\| \int_{0}^{\eta}\left|f\left(x, u(x),{ }^{c} D^{\alpha-1} u(x)\right)\right| d x+\left\|\mu_{7}\right\| \int_{0}^{T}\left|f\left(x, u(x),{ }^{c} D^{\alpha-1} u(x)\right)\right| d x \\
& +\left\|\mu_{8}\right\| \int_{0}^{\eta}\left|K_{2}(\eta, x)\right|\left|f\left(x, u(x),{ }^{c} D^{\alpha-1} u(x)\right)\right| d x \\
& +\left\|\mu_{9}\right\| \int_{0}^{T}\left|K_{2}(T, x)\right| f\left(x, u(x),{ }^{c} D^{\alpha-1} u(x)\right) \mid d x .
\end{aligned}
$$


But

$$
\begin{aligned}
\left|f\left(t, u(t),{ }^{c} D^{\alpha-1} u(t)\right)\right| & =\left|f\left(t, u(t),{ }^{c} D^{\alpha-1} u(t)\right)-f(t, 0,0)+f(t, 0,0)\right| \\
& \leq\left|f\left(t, u(t),{ }^{c} D^{\alpha-1} u(t)\right)-f(t, 0,0)\right|+|f(t, 0,0)| \\
& \leq L_{f}\|u\|_{\alpha-1}+\sup _{0 \leq t \leq T}|f(t, 0,0)| \leq L_{f} r+N_{f} .
\end{aligned}
$$

Then

$$
\begin{aligned}
\|\mathcal{F} u\| \leq & \left\|\mu_{1}\right\|+\left(L_{f} r+N_{f}\right) \\
& \times\left(\frac{T^{\alpha-1}}{k \Gamma(\alpha)}\left(1-e^{-k T}\right)+\left\|\mu_{2}\right\| \int_{0}^{\xi} \frac{s^{\alpha-1}}{k \Gamma(\alpha)}\left(1-e^{-k s}\right) d s\right. \\
& +\left\|\mu_{3}\right\| \int_{\zeta}^{T} \frac{s^{\alpha-1}}{k \Gamma(\alpha)}\left(1-e^{-k s}\right) d s+\left\|\mu_{4}\right\| \frac{\eta^{\alpha-1}}{k \Gamma(\alpha)}\left(1-e^{-k \eta}\right) \\
& +\left\|\mu_{5}\right\| \frac{T^{\alpha-1}}{k \Gamma(\alpha)}\left(1-e^{-k T}\right)+\eta\left\|\mu_{6}\right\|+T\left\|\mu_{7}\right\| \\
& \left.+\left\|\mu_{8}\right\| \frac{\eta\left(1-e^{-k \eta}\right)}{k}+\left\|\mu_{9}\right\| \frac{T\left(1-e^{-k T}\right)}{k}\right) \\
\leq & \left\|\mu_{1}\right\|+\left(L_{f} r+N_{f}\right) \mathcal{R}_{1},
\end{aligned}
$$

and

$$
\begin{aligned}
\left\|{ }^{c} D^{\alpha-1} \mathcal{F} u\right\| \leq & \left\|\left({ }^{c} D^{\alpha-1} \mu_{1}\right)\right\|+\left(L_{f} r+N_{f}\right) \\
& \times\left(T+T\left(1-e^{-k T}\right)+\left\|{ }^{c} D^{\alpha-1} \mu_{2}\right\| \int_{0}^{\xi} \frac{s^{\alpha-1}}{k \Gamma(\alpha)}\left(1-e^{-k s}\right) d s\right. \\
& +\left\|{ }^{c} D^{\alpha-1} \mu_{3}\right\| \int_{\zeta}^{T} \frac{s^{\alpha-1}}{k \Gamma(\alpha)}\left(1-e^{-k s}\right) d s+\left\|{ }^{c} D^{\alpha-1} \mu_{4}\right\| \frac{\eta^{\alpha-1}}{k \Gamma(\alpha)}\left(1-e^{-k \eta}\right) \\
& +\left\|{ }^{c} D^{\alpha-1} \mu_{5}\right\| \frac{T^{\alpha-1}}{k \Gamma(\alpha)}\left(1-e^{-k T}\right)+\eta\left\|{ }^{c} D^{\alpha-1} \mu_{6}\right\|+T\left\|{ }^{c} D^{\alpha-1} \mu_{7}\right\| \\
& \left.+\left\|{ }^{c} D^{\alpha-1} \mu_{8}\right\| \frac{\eta\left(1-e^{-k \eta}\right)}{k}+\left\|{ }^{c} D^{\alpha-1} \mu_{9}\right\| \frac{T\left(1-e^{-k T}\right)}{k}\right) \\
\leq & \left\|{ }^{c} D^{\alpha-1} \mu_{1}\right\|+\left(L_{f} r+N_{f}\right) \mathcal{R}_{2} .
\end{aligned}
$$

Combining (10) and (11) we get

$$
\|\mathcal{F} u\|_{\alpha-1} \leq\left\|\mu_{1}\right\|_{\alpha-1}+\left(L_{f} r+N_{f}\right)\left(\mathcal{R}_{1}+\mathcal{R}_{2}\right)=\left\|\mu_{1}\right\|_{\alpha-1}+\left(L_{f} r+N_{f}\right) \mathcal{R} \leq r,
$$

which implies that $\mathcal{F} \mathfrak{B}_{r} \subset \mathfrak{B}_{r}$.

Next we show that the operator $\mathcal{F}$ is a contraction. $\forall u, v \in \Psi$ we have

$$
\begin{aligned}
& |(\mathcal{F} u)(t)-(\mathcal{F} v)(t)| \\
& \quad \leq \int_{0}^{t}\left|K_{1}(t, x)\right|\left|f\left(x, u(x),{ }^{c} D^{\alpha-1} u(x)\right)-f\left(x, v(x),{ }^{c} D^{\alpha-1} v(x)\right)\right| d x
\end{aligned}
$$




$$
\begin{aligned}
& +\left\|\mu_{2}\right\| \int_{0}^{\xi} \int_{0}^{s}\left|K_{1}(s, x)\right|\left|f\left(x, u(x),{ }^{c} D^{\alpha-1} u(x)\right)-f\left(x, v(x),{ }^{c} D^{\alpha-1} v(x)\right)\right| x d s \\
& +\left\|\mu_{3}\right\| \int_{\zeta}^{T} \int_{0}^{s}\left|K_{1}(s, x)\right|\left|f\left(x, u(x),{ }^{c} D^{\alpha-1} u(x)\right)-f\left(x, v(x),{ }^{c} D^{\alpha-1} v(x)\right)\right| d x d s \\
& +\left\|\mu_{4}\right\| \int_{0}^{\eta}\left|K_{1}(\eta, x)\right| f\left(x, u(x),{ }^{c} D^{\alpha-1} u(x)\right)-f\left(x, v(x),{ }^{c} D^{\alpha-1} v(x)\right) \mid d x \\
& +\left\|\mu_{5}\right\| \int_{0}^{T}\left|K_{1}(T, x)\right|\left|f\left(x, u(x),{ }^{c} D^{\alpha-1} u(x)\right)-f\left(x, v(x),{ }^{c} D^{\alpha-1} v(x)\right)\right| d x \\
& +\left\|\mu_{6}\right\| \int_{0}^{\eta}\left|f\left(x, u(x),{ }^{c} D^{\alpha-1} u(x)\right)-f\left(x, v(x),{ }^{c} D^{\alpha-1} v(x)\right)\right| d x \\
& +\left\|\mu_{7}\right\| \int_{0}^{T}\left|f\left(x, u(x),{ }^{c} D^{\alpha-1} u(x)\right)-f\left(x, v(x),{ }^{c} D^{\alpha-1} v(x)\right)\right| d x \\
& +\left\|\mu_{8}\right\| \int_{0}^{\eta}\left|K_{2}(\eta, x)\right|\left|f\left(x, u(x),{ }^{c} D^{\alpha-1} u(x)\right)-f\left(x, v(x),{ }^{c} D^{\alpha-1} v(x)\right)\right| d x \\
& +\left\|\mu_{9}\right\| \int_{0}^{T}\left|K_{2}(T, x)\right|\left|f\left(x, u(x),{ }^{c} D^{\alpha-1} u(x)\right)-f\left(x, v(x),{ }^{c} D^{\alpha-1} v(x)\right)\right| d x \\
& \leq L \mathcal{R}_{1}\|u-v\|_{\alpha-1} .
\end{aligned}
$$

In a like manner

$$
\left\|{ }^{c} D^{\alpha-1} \mathcal{F} u-{ }^{c} D^{\alpha-1} \mathcal{F} v\right\| \leq L \mathcal{R}_{2}\|u-v\|_{\alpha-1}
$$

From (12) and (13) it follows that

$$
\|\mathcal{F} u-\mathcal{F} v\|_{\alpha-1} \leq L \mathcal{R}\|u-v\|_{\alpha-1}
$$

So $\mathcal{F}$ is a contraction. By the Banach contraction mapping theorem the BVP has a unique solution on $[0, T]$.

The second result is about the existence and uniqueness of solution.

Theorem 2 Assume that (A1), (A2) and (A3) hold. If $L_{f} \mathcal{R}_{3}<1$, then the BVP (1) has a unique solution on $[0, T]$.

Proof Consider the ball $\mathfrak{B}_{r}=\left\{u \in \Psi:\|u\|_{\alpha-1} \leq r\right\}$ with $r \geq\left\|\mu_{1}\right\|_{\alpha-1}+\mathcal{R}\|y\|$. We define two operators $\mathcal{F}_{1}$ and $\mathcal{F}_{2}$ on $\mathfrak{B}_{r}$ as follows:

$$
\begin{aligned}
\left(\mathcal{F}_{1} u\right)(t)= & \int_{0}^{t} K_{1}(t, x) f\left(x, u(x),{ }^{c} D^{\alpha-1} u(x)\right) d x \\
\left(\mathcal{F}_{2} u\right)(t)= & \mu_{1}(t)+\mu_{2}(t) \int_{0}^{\xi} \int_{0}^{s} K_{1}(s, x) f\left(x, u(x),{ }^{c} D^{\alpha-1} u(x)\right) d x d s \\
& +\mu_{3}(t) \int_{\zeta}^{T} \int_{0}^{s} K_{1}(s, x) f\left(x, u(x),{ }^{c} D^{\alpha-1} u(x)\right) d x d s \\
& +\mu_{4}(t) \int_{0}^{\eta} K_{1}(\eta, x) f\left(x, u(x),{ }^{c} D^{\alpha-1} u(x)\right) d x
\end{aligned}
$$




$$
\begin{aligned}
& +\mu_{5}(t) \int_{0}^{T} K_{1}(T, x) f\left(x, u(x),{ }^{c} D^{\alpha-1} u(x)\right) d x \\
& +\mu_{6}(t) \int_{0}^{\eta} f\left(x, u(x),{ }^{c} D^{\alpha-1} u(x)\right) d x \\
& +\mu_{7}(t) \int_{0}^{T} f\left(x, u(x),{ }^{c} D^{\alpha-1} u(x)\right) d x \\
& +\mu_{8}(t) \int_{0}^{\eta} K_{2}(\eta, x) f\left(x, u(x),{ }^{c} D^{\alpha-1} u(x)\right) d x \\
& +\mu_{9}(t) \int_{0}^{T} K_{2}(T, x) f\left(x, u(x),{ }^{c} D^{\alpha-1} u(x)\right) d x .
\end{aligned}
$$

For $u, v \in \mathfrak{B}_{r}$, it is clear that $\left\|\mathcal{F}_{1} u+\mathcal{F}_{2} v\right\|_{\alpha-1} \leq\left\|\mu_{1}\right\|_{\alpha-1}+\mathcal{R}\|y\| \leq r$ thus $\mathcal{F}_{1} u+\mathcal{F}_{2} v \in \mathfrak{B}_{r}$. By using condition (A2), one can also easily show that $\left\|\mathcal{F}_{2} u-\mathcal{F}_{2} v\right\|_{\alpha-1} \leq L \mathcal{R}_{3}\|u-v\|_{\alpha-1}$, which implies that $\mathcal{F}_{2}$ is a contraction.

In addition, the operator $\mathcal{F}_{1}$ is continuous as a result of the continuity of $f$. Also $\mathcal{F}_{1}$ is uniformly bounded as

$$
\begin{aligned}
\left|\left(\mathcal{F}_{1} u\right)(t)\right| & \leq \int_{0}^{t} K_{1}(t, x)\left|f\left(x, u(x),{ }^{c} D^{\alpha-1} u(x)\right)\right| d x \\
& \leq\|y\| \frac{T^{\alpha-1}}{k \Gamma(\alpha)}\left(1-e^{-k T}\right) .
\end{aligned}
$$

Note that

$$
\begin{aligned}
\left({ }^{c} D^{\alpha-1} \mathcal{F}_{1} u\right)(t)= & { }^{c} D^{\alpha-1}\left[\int_{0}^{t} K_{1}(t, x) f\left(x, u(x),{ }^{c} D^{\alpha-1} u(x)\right) d x\right] \\
= & \int_{0}^{t} f\left(x, u(x),{ }^{c} D^{\alpha-1} u(x)\right) d x-k \int_{0}^{t} K_{2}(t, x) f\left(x, u(x),{ }^{c} D^{\alpha-1} u(x)\right) d x \\
\left|\left({ }^{c} D^{\alpha-1} \mathcal{F}_{1} u\right)(t)\right| \leq & \int_{0}^{t}\left|f\left(x, u(x),{ }^{c} D^{\alpha-1} u(x)\right)\right| d x \\
& +k \int_{0}^{t} K_{2}(t, x)\left|f\left(x, u(x),{ }^{c} D^{\alpha-1} u(x)\right)\right| d x \\
\leq & \|y\| T+\frac{T^{\alpha-1}}{\Gamma(\alpha)}\left(1-e^{-k T}\right) .
\end{aligned}
$$

As $0 \leq t \leq T$, from (14) and (15) we obtain

$$
\left\|\mathcal{F}_{1} u\right\|_{\alpha-1} \leq\|y\|\left(T+\frac{T^{\alpha-1}}{k \Gamma(\alpha)}\left(1-e^{-k T}\right)+\frac{T^{\alpha-1}}{\Gamma(\alpha)}\left(1-e^{-k T}\right)\right)
$$

Now it will be shown that the operator $\mathcal{F}_{1}$ is compact. We fix $\sup _{(t, u, v) \in[0, T] \times \mathfrak{B}_{r} \times \mathfrak{B}_{r}} \mid f(t$, $u, v) \mid=f_{r}$.

For $t_{1}<t_{2}, t_{1}, t_{2} \in[0, T]$, then

$$
\begin{aligned}
& \left|\mathcal{F}_{1} u\left(t_{2}\right)-\mathcal{F}_{1} u\left(t_{1}\right)\right| \\
& \quad \leq\left|\int_{0}^{t_{1}} K_{1}\left(t_{2}, x\right)-K_{1}\left(t_{1}, x\right) d x\right|+\left|\int_{t_{1}}^{t_{2}} K_{1}\left(t_{2}, x\right) d x\right|
\end{aligned}
$$




$$
\begin{aligned}
\leq & \left|e^{-k t_{2}}-e^{-k t_{1}}\right| \int_{0}^{t_{1}} e^{k x}\left|I^{\alpha-1} f\left(x, u(x),{ }^{c} D^{\alpha-1} u(x)\right)\right| d x \\
& +\int_{t_{1}}^{t_{2}} e^{-k\left(t_{2}-x\right)}\left|I^{\alpha-1} f\left(x, u(x),{ }^{c} D^{\alpha-1} u(x)\right)\right| d x \\
\leq & \|y\|\left(\left|e^{-k t_{2}}-e^{-k t_{1}}\right| \int_{0}^{t_{1}} e^{k x}\left|I^{\alpha-1} 1(x)\right| d x+\int_{t_{1}}^{t_{2}} e^{-k\left(t_{2}-x\right)}\left|I^{\alpha-1} 1(x)\right| d x\right) \rightarrow 0,
\end{aligned}
$$

as $t_{2}-t_{1} \rightarrow 0$. In a like manner

$$
\left|{ }^{c} D^{\alpha-1} \mathcal{F}_{1} u\left(t_{2}\right)-{ }^{c} D^{\alpha-1} \mathcal{F}_{1} u\left(t_{1}\right)\right| \rightarrow 0 \quad \text { as } t_{2} \rightarrow t_{1} .
$$

Equations (16) and (17) imply that $\mathcal{F}_{1}$ is relatively compact, and by the Arzela-Ascoli theorem we conclude that $\mathcal{F}_{1}$ is compact on $\mathfrak{B}_{r}$. Hence, the existence of the solution of the BVP holds by Krasnoselskii's fixed point theorem.

Theorem 3 Assume that (A1), (A4) and (A5) hold. Then the BVP (1) has at least one solution on $[0, T]$.

Proof The proof will be split into several steps. In the first step, we show that the operator $\mathcal{F}: \Psi \rightarrow \Psi$ maps bounded sets into bounded sets of $\Psi$.

Let $\mathfrak{B}_{r}=\left\{u \in \Psi:\|u\|_{\alpha-1} \leq r\right\}$ be a bounded set in $\Psi$. Then by (A4) we have

$$
\begin{aligned}
|(\mathcal{F} u)(t)| \leq & \left\|\mu_{1}\right\|+\int_{0}^{t}\left|K_{1}(t, x)\right| g(x) \chi\left(\|u\|_{\alpha-1}\right) d x \\
& +\left\|\mu_{2}\right\| \int_{0}^{\xi} \int_{0}^{s}\left|K_{1}(s, x)\right| g(x) \chi\left(\|u\|_{\alpha-1}\right) d x d s \\
& +\left\|\mu_{3}\right\| \int_{\zeta}^{T} \int_{0}^{s}\left|K_{1}(s, x)\right| g(x) \chi\left(\|u\|_{\alpha-1}\right) d x d s \\
& +\left\|\mu_{4}\right\| \int_{0}^{\eta}\left|K_{1}(\eta, x)\right| g(x) \chi\left(\|u\|_{\alpha-1}\right) d x \\
& +\left\|\mu_{5}\right\| \int_{0}^{T}\left|K_{1}(T, x)\right| g(x) \chi\left(\|u\|_{\alpha-1}\right) d x \\
& +\left\|\mu_{6}\right\| \int_{0}^{\eta} g(x) \chi\left(\|u\|_{\alpha-1}\right) d x+\left\|\mu_{7}\right\| \int_{0}^{T} g(x) \chi\left(\|u\|_{\alpha-1}\right) d x \\
& +\left\|\mu_{8}\right\| \int_{0}^{\eta}\left|K_{2}(\eta, x)\right| g(x) \chi\left(\|u\|_{\alpha-1}\right) d x \\
& +\left\|\mu_{9}\right\| \int_{0}^{T}\left|K_{2}(T, x)\right| g(x) \chi\left(\|u\|_{\alpha-1}\right) d x .
\end{aligned}
$$

Taking $\sup _{0 \leq t \leq T}$ implies that

$$
\sup _{0 \leq t \leq T}|\mathcal{F} u(t)| \leq\left\|\mu_{1}\right\|+\|g\| \chi(r) \mathcal{R}_{1} .
$$

In a like manner

$$
\sup _{0 \leq t \leq T}\left|{ }^{c} D^{\alpha-1} \mathcal{F} u(t)\right| \leq\left\|{ }^{c} D^{\alpha-1} \mu_{1}\right\|+\|g\| \chi(r) \mathcal{R}_{2} .
$$


Combining (18) and (19) we get

$$
\|\mathcal{F} u\|_{\alpha-1} \leq\left\|\mu_{1}\right\|_{\alpha-1}+\|g\| \chi(r) \mathcal{R} .
$$

Next we show that the operator $\mathcal{F}: \Psi \rightarrow \Psi$ maps bounded sets into equicontinuous sets of $\Psi$. Let $t_{1}, t_{2} \in[0, T]$ with $t_{1}<t_{2}$ and $u \in \mathfrak{B}_{r}$; then we obtain

$$
\begin{aligned}
& \left|(\mathcal{F} u)\left(t_{2}\right)-(\mathcal{F} u)\left(t_{1}\right)\right| \\
& \leq\left|\mu_{1}\left(t_{2}\right)-\mu_{1}\left(t_{1}\right)\right| \\
& \quad+\left|\int_{0}^{t_{1}} K_{1}\left(t_{1}, x\right) g(x) \chi\left(\|u\|_{\alpha-1}\right) d x-\int_{0}^{t_{2}} K_{1}\left(t_{2}, x\right) g(x) \chi\left(\|u\|_{\alpha-1}\right) d x\right| \\
& \quad+\left|\mu_{2}\left(t_{2}\right)-\mu_{2}\left(t_{1}\right)\right| \int_{0}^{\xi} \int_{0}^{s}\left|K_{1}(s, x)\right| g(x) \chi\left(\|u\|_{\alpha-1}\right) d x d s \\
& \quad+\left|\mu_{3}\left(t_{2}\right)-\mu_{3}\left(t_{1}\right)\right| \int_{\zeta}^{T} \int_{0}^{s}\left|K_{1}(s, x)\right| g(x) \chi\left(\|u\|_{\alpha-1}\right) d x d s \\
& \quad+\left|\mu_{4}\left(t_{2}\right)-\mu_{4}\left(t_{1}\right)\right| \int_{0}^{\eta}\left|K_{1}(\eta, x)\right| g(x) \chi\left(\|u\|_{\alpha-1}\right) d x+\mid \mu_{5}\left(t_{2}\right) \\
& \quad-\mu_{5}\left(t_{1}\right)\left|\int_{0}^{T}\right| K_{1}(T, x) \mid g(x) \chi\left(\|u\|_{\alpha-1}\right) d x \\
& \quad+\left|\mu_{6}\left(t_{2}\right)-\mu_{6}\left(t_{1}\right)\right| \int_{0}^{\eta} g(x) \chi\left(\|u\|_{\alpha-1}\right) d x+\left|\mu_{7}\left(t_{2}\right)-\mu_{7}\left(t_{1}\right)\right| \int_{0}^{T} g(x) \chi\left(\|u\|_{\alpha-1}\right) d x \\
& \quad+\left|\mu_{8}\left(t_{2}\right)-\mu_{8}\left(t_{1}\right)\right| \int_{0}^{\eta}\left|K_{2}(\eta, x)\right| g(x) \chi\left(\|u\|_{\alpha-1}\right) d x+\mid \mu_{9}\left(t_{2}\right) \\
& \quad-\mu_{9}\left(t_{1}\right)\left|\int_{0}^{T}\right| K_{2}(T, x) \mid g(x) \chi\left(\|u\|_{\alpha-1}\right) d x .
\end{aligned}
$$

Then the right hand side of the above inequality tends to zero as $t_{1} \rightarrow t_{2}$. That is $\mid \mathcal{F} u\left(t_{2}\right)-$ $\mathcal{F} u\left(t_{1}\right) \mid \rightarrow 0$ as $t_{1} \rightarrow t_{2}$. In a like manner

$$
\left|{ }^{c} D^{\alpha-1} \mathcal{F} u\left(t_{2}\right)-{ }^{c} D^{\alpha-1} \mathcal{F} u\left(t_{1}\right)\right| \rightarrow 0 \quad \text { as } t_{1} \rightarrow t_{2} .
$$

Note that the right hand side of the above inequality is independent of $u \in \mathfrak{B}_{r}$, by ArzelaAscoli theorem we conclude that $\mathcal{F}$ is completely continuous.

The last step to complete the assumptions of the Leray-Schauder nonlinear alternative theorem is to show the boundedness of the set of all solution to equation $u=\delta \mathcal{F} u, \delta \in$ $[0,1]$. Assume that $u$ is a solution, Then in the same manner as we show the operator $\mathcal{F}$ is bounded, we can obtain

$$
|u(t)|=|\delta(\mathcal{F} u)(t)| \leq \delta\left(\left\|\mu_{1}\right\|_{\alpha-1}+\|g\| \chi(r) \mathcal{R}\right) \leq\left\|\mu_{1}\right\|_{\alpha-1}+\|g\| \chi(r) \mathcal{R} .
$$

This implies

$$
\frac{\|u\|}{\left\|\mu_{1}\right\|_{\alpha-1}+\|g\| \chi(r) \mathcal{R}} \leq 1
$$

But by (A5) there exists a constant such that $W \neq u$. 
Construct the set $\Omega=\{u \in \phi: u<W\}$. It is obvious that the operator $\mathcal{F}: \bar{\Omega} \rightarrow \Psi$ is continuous and completely continuous, by the constructed $\Omega, \nexists u \in \partial \Omega$ such that $u=\delta \mathcal{F}_{u}$ for some $\delta \in(0,1)$. Consequently, by the nonlinear alternative of Leray-Schauder type, we deduce that $\mathcal{F}$ has a fixed point $u \in \bar{\Omega}$ which is a solution of the BVP.

\section{Examples}

Given the problem

$$
\left\{\begin{array}{l}
\left({ }^{c} D^{3 / 2}+{ }^{c} D^{1 / 2}\right) u(t)=L\left(\frac{u(t)}{1+u(t)}+\tan ^{-1}\left({ }^{c} D^{1 / 2} u(t)\right)+\sqrt{\sin ^{3} t}\right), \quad 0 \leq t \leq 3, \\
u(0)+u(3)=\int_{0}^{1} u(s) d s, \quad{ }^{c} D^{1 / 2} u(0)+{ }^{c} D^{1 / 2} u(3)=\int_{0}^{1} u(s) d s+1 .
\end{array}\right.
$$

Here

$$
\begin{aligned}
& f\left(t, u(t),{ }^{c} D^{1 / 2} u(t)\right)=L\left(\frac{u(t)}{1+u(t)}+\tan ^{-1}\left({ }^{c} D^{1 / 2} u(t)\right)+\sqrt{\sin ^{3} t}\right), \\
& k=1, \quad \alpha_{1}=\alpha_{2}=\beta_{1}=\beta_{2}=\gamma_{1}=\gamma_{2}=1, \\
& \eta=0, \quad \xi=1, \quad \zeta=2, \quad T=3, \quad \varepsilon_{1}=0, \quad \varepsilon_{2}=1, \\
& q=\left(\alpha_{1} e^{-k \eta}+\beta_{1} e^{-k T}-\gamma_{1}\left(1-e^{-k \xi}\right)\right)=0.42, \\
& \rho=\left(\alpha_{1}+\beta_{1}-\gamma_{1} \xi\right)=1, \\
& \sigma=\left(\frac{\gamma_{2}}{k}\left(e^{-k \zeta}-e^{-k T}\right)+\frac{k \alpha_{2}}{\Gamma(2-\alpha)} \int_{0}^{\eta}(\eta-s)^{1-\alpha} e^{-k s} d s+\frac{k \beta_{2}}{\Gamma(2-\alpha)} \int_{0}^{T}(T-s)^{1-\alpha} e^{-k s} d s\right) \\
& \leq 2.04, \\
& z=\gamma_{2}(T-\zeta)-\frac{\sigma \rho}{q}=-3.86, \\
& \left\|\mu_{2}\right\|=\left|\left(\frac{\gamma_{1} \rho \sigma}{q^{2} z}+\frac{\gamma_{1}}{q}\right)-\frac{\gamma_{1} \sigma}{q z}\right|=6.86, \\
& \left\|\mu_{3}\right\|=\left|\left(\frac{\gamma_{2} \rho}{q z}\right)-\left(\frac{\gamma_{2}}{z}\right)\right|=0.36, \\
& \left\|\mu_{4}\right\|=\left|\left(\frac{\alpha_{1} \sigma}{q z}\right)-\left(\frac{\alpha_{1} \rho \sigma}{q^{2} z}+\frac{\alpha_{1}}{q}\right) e^{-k T}\right|=1.23, \\
& \left\|\mu_{8}\right\|=\left|\left(\frac{\alpha_{2} k \rho}{q z}\right)-\frac{\alpha_{2} k}{z}\right|=0.36, \\
& \left\|\mu_{5}\right\|=\left|\left(\frac{\beta_{1} \sigma}{q z}\right)-\left(\frac{\beta_{1} \rho \sigma}{q^{2} z}+\frac{\beta_{1}}{q}\right) e^{-k T}\right|=1.23, \\
& \left\|\mu_{7}\right\|=\left|\frac{\alpha_{2}}{z}-\left(\frac{\beta_{2} \rho}{q z}\right) e^{-k T}\right|=0.23, \\
& \left.\| \frac{\beta_{2} \rho}{q z}\right) e^{-k T} \mid=0.23, \\
& \| \mu_{2},
\end{aligned}
$$


Clearly $f$ is continuous and $\forall t \in[0,2], \forall u, v, \bar{u}, \bar{v} \in \mathbb{R}$ we have

$$
|f(t, u, v)-f(t, \bar{u}, \bar{v})| \leq L\left|\frac{|u|}{1+|u|}-\frac{|\bar{u}|}{1+|\bar{u}|}\right|+L\left|\tan ^{-1} v-\tan ^{-1} \bar{v}\right| \leq L(|u-\bar{u}|+|v-\bar{v}|),
$$

with $\mathcal{R}_{1} \leq 11.25, \mathcal{R}_{2} \leq 21.72, \mathcal{R}_{3} \leq 18.17$

Choosing $L<\frac{1}{\mathcal{R}_{1}+\mathcal{R}_{2}}$, Theorem 1 implies that our problem has unique solution.

For the illustration of Theorem 3 consider the function

$$
\begin{aligned}
& f\left(t, u(t),{ }^{c} D^{1 / 2} u(t)\right)=t^{2} e^{-u^{2}(t)} \ln \left(1+2 \sin ^{2} u(t)\right)+\frac{{ }^{c} D^{3 / 2} u(t)}{1+{ }^{c} D^{3 / 2} u(t)}+\sqrt{1+t^{2}}, \\
& f(t, u, v)=t^{2} e^{-u^{2}} \ln \left(1+2 \sin ^{2} u\right)+\frac{|v|}{1+|v|}+\sqrt{1+t^{2}}, \quad t \in[0,3], u, v \in \mathbb{R}
\end{aligned}
$$

clearly $f$ is continuous and bounded as

$$
|f(t, u, v)| \leq t^{2} \ln 3+1+\sqrt{1+t^{2}}=: g(t), \quad \text { with }\|g\|=9(\ln 3)+1+\sqrt{10},
$$

$g(t) \in C_{1 / 2}[0,3]$, and then the BVP has a solution in [0,3].

For the applicability of Theorem 2 consider the function

$$
f\left(t, u(t),{ }^{c} D^{1 / 2} u(t)\right)=L\left(\frac{u(t)}{1+u(t)}+\tan ^{-1}\left({ }^{c} D^{1 / 2} u(t)\right)+\sqrt{\sin ^{3} t}\right),
$$

where $f$ is continuous, Lipschitzian and bounded as

$$
|f(t, u, v)| \leq L\left(1+\frac{\pi}{2}+\sqrt{\sin ^{3} t}\right)=: y(t) \leq L\left(2+\frac{\pi}{2}\right),
$$

with $\mathcal{R}_{3} \leq 11.25$; if we choose $L<\frac{1}{\mathcal{R}_{3}}$, then the BVP has a solution in [0,3].

\section{Concluding remarks}

In this paper, we have discussed the existence and uniqueness of solutions for a new class of boundary value problems consisting of sequential fractional differential equations supplemented with four point nonlocal integral fractional boundary conditions. It should be stressed that, similarly problems for nonlocal separated (non separated) multi-point boundary value problems can be investigated. So the present work is a useful contribution to the existing literature on the topic.

\section{Acknowledgements}

The authors thank the reviewers for their useful comments, which led to the improvement of the original manuscript.

Competing interests

The authors declare that they have no competing interests.

Authors' contributions

Each of the authors, NIM, MA, and KA contributed to each part of this work equally and read and approved the final version of the manuscript.

\section{Publisher's Note}

Springer Nature remains neutral with regard to jurisdictional claims in published maps and institutional affiliations. 


\section{References}

1. Podlubny, I: Fractional Differential Equations. Academic Press, San Diego (1999)

2. Kilbas, AA, Srivastava, HM, Trujillo, JJ: Theory and Applications of Fractional Differential Equations. North-Holland Mathematics Studies, vol. 204. Elsevier, Amsterdam (2006)

3. Yang, X-J, Baleanu, D, Srivastava, HM: Local Fractional Integral Transforms and Their Applications

4. Almeida, R, Malinowska, AB, Odzijewicz, T: Fractional differential equations with dependence on the Caputo-Katugampola derivative. J. Comput. Nonlinear Dyn. 11(6), 061017 (2016)

5. Alsaedi, A, Baleanu, D, Etemad, S, Rezapour, S: On coupled systems of time-fractional differential problems by using a new fractional derivative. J. Funct. Spaces (2016)

6. Alkahtani, BST, Atangana, A: Controlling the wave movement on the surface of shallow water with the Caputo-Fabrizio derivative with fractional order. Chaos Solitons Fractals 89, 539-546 (2016)

7. Atangana, A, Baleanu, D: Caputo-Fabrizio derivative applied to groundwater flow within confined aquifer. J. Eng. Mech. 143(5), D4016005 (2017)

8. Atangana, A, Alkahtani, BST: New model of groundwater flowing within a confine aquifer: application of Caputo-Fabrizio derivative. Arab. J. Geosci. 9(1), 8 (2016)

9. Atangana, A, Baleanu, D: New fractional derivatives with non-local and non-singular kernel: theory and application to heat transfer model. Therm. Sci. 20(2), 763-769 (2016)

10. Gao, F, Yang, X-J: Fractional Maxwell fluid with fractional derivative without singular kernel. Therm. Sci. 20, S871-S877 (2016)

11. Mahmudov, NI: Finite-approximate controllability of evolution equations. Appl. Comput. Math. 16(2), 159-167 (2017)

12. Mahmudov, NI, McKibben, MA: On approximately controllable systems (survey). Appl. Comput. Math. 15(3), 247-264 (2016)

13. Marin, M: On weak solutions in elasticity of dipolar bodies with voids. J. Comput. Appl. Math. 82(1-2), 291-297 (1997)

14. Marin, M: Harmonic vibrations in thermoelasticity of microstretch materials. J. Vib. Acoust. 132(4), 044501 (2010)

15. Sharma, K, Marin, M: Effect of distinct conductive and thermodynamic temperatures on the reflection of plane waves in micropolar elastic half-space. Sci. Bull. "Politeh." Univ. Buchar., Ser. A, Appl. Math. Phys. 75(2), 121-132 (2013)

16. Yang, X-J: Fractional derivatives of constant and variable orders applied to anomalous relaxation models in heat-transfer problems. Therm. Sci. 2017(3), 1161-1171 (2016)

17. Yang, X-J, Machado, JAT: A new fractional operator of variable order: application in the description of anomalous diffusion. Phys. A, Stat. Mech. Appl. 481, 276-283 (2017)

18. Yang, X-J, Srivastava, HM, Machado, JAT: A new fractional derivative without singular kernel: application to the modelling of the steady heat flow. Therm. Sci. 20(2), 753-756 (2016)

19. Ahmad, B, Nieto, JJ: Sequential fractional differential equations with three-point boundary conditions. Comput. Math Appl. 64, 3046-3052 (2012)

20. Ahmad, B, Nieto, JJ: Boundary value problems for a class of sequential integrodifferential equations of fractional order. J. Funct. Spaces Appl. 2013, Article ID 149659 (2013)

21. Ahmad, B, Ntouyas, SK: Existence results for a coupled system of Caputo type sequential fractional differential equations with nonlocal integral boundary conditions. Appl. Math. Comput. 266, 615-622 (2015)

22. Aqlan, MH, Alsaedi, A, Ahmad, B, Nieto, JJ: Existence theory for sequential fractional differential equations with anti-periodic type boundary conditions. Open Math. 14, 723-735 (2016)

23. Klimek, M: Sequential fractional differential equations with Hadamard derivative. Commun. Nonlinear Sci. Numer Simul. 16, 4689-4697 (2011)

24. Ye, H, Huang, R: On the nonlinear fractional differential equations with Caputo sequential fractional derivative. Adv. Math. Phys. 2015, Article ID 174156 (2015)

25. Ahmad, B, Ntouyas, SK: A higher-order nonlocal three-point boundary value problem of sequential fractional differential equations. Miskolc Math. Notes 15(2), 265-278 (2014)

26. Ahmad, B, Losada, J, Nieto, JJ: On antiperiodic nonlocal three-point boundary value problems for nonlinear fractiona differential equations. Discrete Dyn. Nat. Soc. 2015, Article ID 973783 (2015)

27. Mahmudov, NI, Unul, S: On existence of BVP's for impulsive fractional differential equations. Adv. Differ. Equ. 2017, 15 (2017)

28. Mahmudov, $\mathrm{NI}$, Unul, S: Existence of solutions of $\alpha \in(2,3]$ order fractional three-point boundary value problems with integral conditions. Abstr. Appl. Anal. 2014, Article ID 198632 (2014)

29. Mahmudov, NI, Mahmoud, H: Four-point impulsive multi-orders fractional boundary value problems. J. Comput. Anal. Appl. 22(7), 1249-1260 (2017)

30. Atangana, A, Koca, I: On the new fractional derivative and application to nonlinear Baggs and Freedman model. J. Nonlinear Sci. Appl. 9, 2467-2480 (2016) 